\title{
A PHYLOGENETIC LIST OF THE GENERA OF RUBIACEAE IN MEXICO
}

\author{
DAVID H. LORENCE \\ National Tropical Botanical Garden \\ P. O. Box 340 , \\ Lawai, Kauai, HI 96765 USA
}

\begin{abstract}
RESUMEN
La familia Rubiaceae está bien representada en la República Mexicana, con 77 géneros indígenas o endémicos, ocho géneros introducidos (naturalizados o cultivados), y aproximadamente 533 especies. Se presenta una secuencia filogenética para ubicar estos géneros según el sistema de clasificación elaborado por Robbrecht (1988).
\end{abstract}

\begin{abstract}
The Rubiaceae are extremely well represented in the Mexican Republic, with 77 indigenous or endemic genera, eight introduced (naturalized or cultivated) genera, and approximately 533 species. A phylogenetic sequence is presented to accommodate these genera following the system of classification proposed by Robbrecht (1988).
\end{abstract}

The Rubiaceae are the world's fourth largest angiosperm family, with approximately 637 genera and 10,700 species (Mabberley, 1987). The family is extremely well represented in the Mexican Republic, where I currently recognize 77 indigenous or endemic and eight introduced genera, comprising approximately 533 species (Appendix I). These figures are based on ten years experience studying the systematics and floristics of Mexican Rubiaceae. In spite of the floristic importance of the Rubiaceae in Mexico (and Central America), there are no recent publications on the phylogeny of the Mexican genera. The only existing floristic studies of Mexican Rubiaceae are Standley's now outdated treatments in Trees and Shrubs of Mexico (Standley, 1926) and the North American Flora (Standley, 1918, 1921, 1934a, 1934b), which is about two-thirds complete for the family. In the former work Standley adopted an alphabetical order for the genera, whereas in the latter he omitted the subfamilies but closely followed the traditional but now antiquated tribal classifications of Hooker (1873) and Schumann (1891). Authors of the only recent floristic treatments for countries in the Central American region, i.e., Guatemala (Standley \& Williams, 1975) and Panama (Dwyer, 1980), have followed Standley's original tribal concepts. The only other phylogeny proposed for the Neotropical Rubiaceae is that of Garcia-Kirkbride (1982) for the Cundinamarca region of Colombia, which encompasses only a part of the Mexican genera.

Although no additional comprehensive floristic treatments for Rubiaceae exist for Mexico or Central America, work is currently in progress on a number of projects that are due to be published in the near future. These include the following: Flora Mesoamericana (Dwyer \& Lorence, in preparation), Flora de Nicaragua (Dwyer, in preparation), Flora de 
Veracruz (Lorence \& Castillo-Campos, in preparation), Flora of Chiapas (Lorence \& Breedlove, in preparation), Flora of Costa Rica (Burger, in preparation), and Manual to the Plants of Costa Rica (Taylor \& Hammel, in preparation).

The following phylogenetic sequence of the genera of Rubiaceae occurring in Mexico follows the most natural and modern classification available, that of Robbrecht (1988), who not only synthesized the vast amount of existing information on morphology and anatomy, but also incorporated many new data, for example from studies of chemistry and pollen. $\mathrm{He}$ also gives an excellent synopsis and evaluation of existing classification schemes. Robbrecht's classification recognizes four subfamilies, the Cinchonoideae, Ixoroideae, Antirheoideae, and Rubioideae, on the basis of character combinations and trends in placentation, floral biology, and morphology and anatomy of fruit and seed. The order in which his tribes appear has been adopted here, but the numbering is different as not all are represented in Mexico. On the basis of my own knowledge of the Mexican Rubiaceae, I choose not to recognize certain genera listed by Robbrecht (1988), i.e. Arachnothryx Planchon, Otocalyx Brandegee, Rogiera Planchon (all three here included in Rondeletia), and Syringantha Standley (here included in Exostema). The rationale for this will be published elsewhere. On the other hand, I recognize Plocaniophyllon, a genus not listed by Robbrecht, and place Eizia in the Hamelieae rather than in the Rondeletieae as he does (for details see Lorence \& Dwyer, 1988). It should be kept in mind that the following sequence is preliminary and may require modification as additional information becomes available. Genera preceded by an asterisk (*) are introduced, either naturalized or commonly cultivated. Only certain generic names still in current use are included as synonyms.

\section{MEXICAN RUBIACEAE GENERA IN A PHYLOGENETIC SYSTEM}

Subfamily 1. Cinchonoideae Rafinesque

Tribe 1. Cinchoneae de Candolle Genera:

Alseis Schott in Sprengel

Balmea M. Martínez

Blepharidium Standley

Bouvardia Salisbury

Calycophyllum de Candolle

Cigarrilla Aiello

Cinchona Linnaeus

Cosmibuena Ruiz \& Pavón

Coutarea Aublet

Exostema (Persoon) Richard ex Humboldt \& Bonpland (incl. Syringantha Standley)

Manettia Mutis ex Linnaeus

Tribe 2. Hillieae Bremekamp ex S. Darwin Genera:

$$
\text { Hillia Jacquin }
$$


Tribe 3. Rondeletieae de Candolle ex Miquel

Genera:

Elaeagia Weddell

Habroneuron Standley

Lindenia Bentham

Rondeletia Linnaeus (incl. Arachnothryx Planchon, Otocalyx

Brandegee \& Rogiera Planchon)

Simira Aublet

Steyermarkia Standley

Tribe 4. Condamineeae J. D. Hooker

Genera:

Pogonopus Klotzsch

Tribe 5. Isertieae A. Richard ex de Candolle

Genera:

Gonzalagunia Ruiz \& Pavón

Isertia Schreber

* Mussaenda Linnaeus

Sabicea Aublet

Subfamily 2. Ixoroideae Rafinesque

Tribe 1. Gardenieae A. Richard ex de Candolle

Genera:

Alibertia A. Richard

Amaioua Aublet

* Gardenia Linnaeus

Genipa Linnaeus

Glossostipula Lorence

Posoqueria Aublet

Randia Linnaeus

Tribe 2. Pavetteae A. Richard ex Dumortier

Genera:

* Ixora Linnaeus

Tribe 3. Coffeeae de Candolle

Genera:

* Coffea Linnaeus

Subfamily 3. Antirheoideae Rafinesque

Tribe 1. Guettardeae de Candolle

Genera:

Antirhea Commerson ex Jussieu

Chomelia Jacquin (incl. Anisomeris Presl)

Guettarda Linnaeus

Machaonia Humboldt \& Bonpland 
Tribe 2. Chiococceae J. D. Hooker Genera:
Allenanthus Standley
Asemnantha J. D. Hooker (as Asemnanthe)
Chiococca P. Browne
Chione de Candolle
Erithalis P. Browne
Placocarpa J. D. Hooker

Tribe 3. Cephalantheae Kunth ex Ridsdale

Genera:

Cephalanthus Linnaeus

Subfamily 4. Rubioideae

Tribe 1. Hedyotideae Chamisso \& Schlechtendal

Genera:

* Dentella J. R. \& G. Forster

Hedyotis Linnaeus (incl. Carterella Terrell, Houstonia

Linnaeus \& Oldenlandia Linnaeus)

* Pentas Bentham Rachicallis de Candolle

Tribe 2. Coccocypseleae Bremekamp

Genera:

Coccocypselum P. Browne

Tribe 3. Hamelieae de Candolle

Genera:

Deppea Chamisso \& Schlechtendal (incl. Edithea Standley)

Eizia Standley

Hamelia Jacquin

Hoffmannia Swartz

Omiltemia Standley

Pinarophyllon Brandegee

Plocaniophyllon Brandegee

Tribe 4. Psychotrieae A. Richard ex Dumortier

Genera:

Cephaelis Swartz

Declieuxia Kunth

Geophila D. Don

Palicourea Aublet

Psychotria Linnaeus

Rudgea R. A. Salisbury

Tribe 5. Morindeae Miquel

Genera:

Appunia J. D. Hooker

Morinda Linnaeus 
Tribe 6. Coussareeae J. D. Hooker

Genera:

Coussarea Aublet

Faramea Aublet

Tribe 7. Paederieae de Candolle

Genera:

Paederia Linnaeus

Tribe 8. Anthospermeae Chamisso \& Schlechtendal

Genera:

Nertera Banks \& Solander ex Gaertner

Tribe 9. Spermacoceae A. Richard ex Dumortier

Genera:

Crusea Schlechtendal \& Chamisso

Diodia Linnaeus

Ernodea Swartz

Mitracarpus Zuccarini

Richardia Linnaeus

Spermacoce Linnaeus (incl. Borreria G. Meyer \& Hemidiodia K. Schumann)

Staelia Chamisso \& Schlechtendal

Tribe 10. Rubieae

Genera:

Didymaea J. D. Hooker

Galium Linnaeus (incl. Relbunium (Endlicher) J. D. Hooker)

* Sherardia Linnaeus

Tribe of uncertain status: Hippotieae García-Kirkbride

Genera:

Sommera Schlechtendal

Genera of uncertain tribal placement:

Bertiera Aublet

Cosmocalyx Standley

Coutaportla Urban

Hintonia Bullock

Mitchella Linnaeus

Strumpfia Jacquin

Dubious genus:

Stylosiphonia Brandegee

Berghesia Nees 
Appendix I. List of genera of Rubiaceae occurring in Mexico (estimated number of species, excluding infraspecific categories, in parentheses; " indicates introduced genera, either cultivated or naturalized).

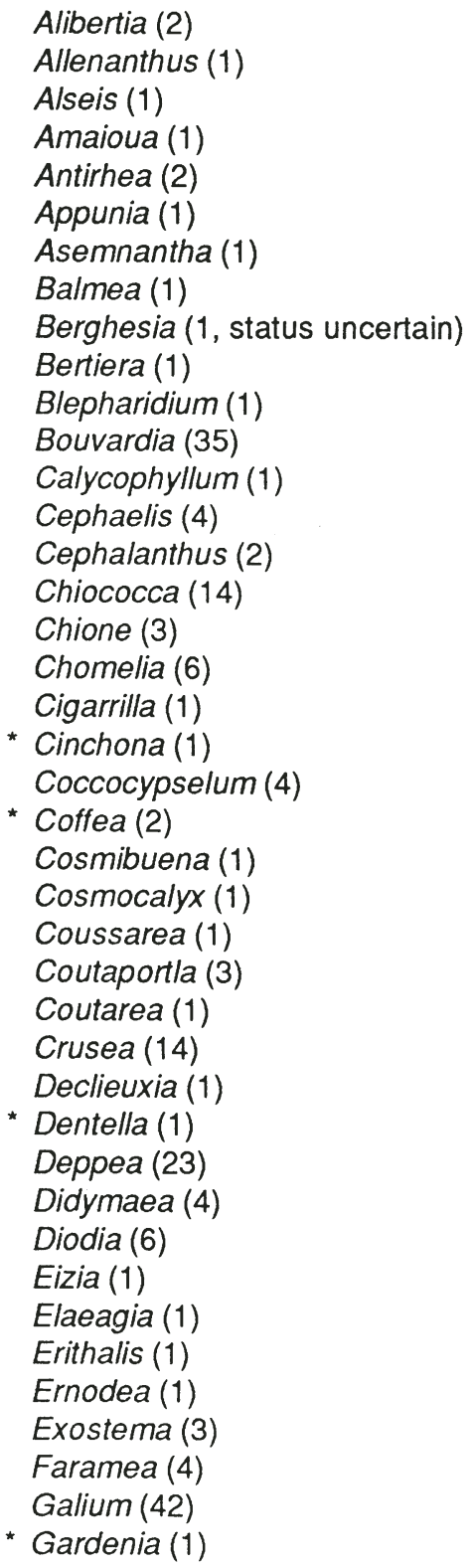

Genipa (1)

Geophila (2)

Glossostipula (2)

Gonzalagunia (3)

Guettarda (10)

Habroneuron (1)

Hamelia (8)

Hedyotis (33)

Hillia (4)

Hintonia (3)

Hoffmannia (ca. 33)

Isertia (1)

* Ixora (3)

Lindenia (1)

Machaonia (7)

Manettia (3)

Mitchella (1)

Mitracarpus (4)

Morinda (4)

* Mussaenda (1)

Nertera (1)

Omiltemia (2)

Paederia (1)

Palicourea (6)

* Pentas (1)

Pinarophyllon (2)

Placocarpa (1)

Plocaniophyllon (1)

Pogonopus (1)

Posoqueria (2)

Psychotria (60)

Rachicallis (1)

Randia (34)

Richardia (5)

Rondeletia (55)

Rudgea (1)

Sabicea (3)

* Sherardia(1)

Simira (4)

Sommera (6)

Spermacoce (ca. 20) 
Staelia (1)

Steyermarkia (1)
Strumpfia (1)

Stylosiphonia (1)

\section{LITERATURE CITED}

Dwyer, J. D. 1980. Rubiaceae. In: Woodson, R. E., Jr. \& R. W. Schery (eds.). Flora of Panama. Ann. Missouri Bot. Gard. 67: 1-522.

Garcia-Kirkbride, M. C. 1982. A preliminary phylogeny for the Neotropical Rubiaceae. PI. Syst. Evol. 141: 115-121.

Hooker, J. D. 1873. Ordo LXXXIV. Rubiaceae. In: Bentham, G. \& J. D. Hooker. Genera Plantarum 2: 7-151. London, Reeve \& Co.

Lorence, D. H. \& J. D. Dwyer. 1988. A revision of Deppea (Rubiaceae). Allertonia 4(7): 389-436.

Mabberley, D. J. 1987. The plant book. Cambridge University Press. Cambridge. 706 pp.

Robbrecht, E. 1988. Tropical woody Rubiaceae. Opera Botanica Belgica 1: 1-271.

Schumann, K. 1891. Rubiaceae. In: Engler, A. \& K. Prantl (eds.). Die natürlichen Pflanzenfamilien 4(4): 1-156. Engelmann. Leipzig.

Standley, P. C. 1918. Rubiaceae. North American Flora 32(1): 1-86.

Standley, P. C. 1921. Rubiaceae. North American Flora 32(2): 87-158.

Standley, P. C. 1926. Rubiaceae. Trees and shrubs of Mexico. Contr. U. S. Nat. Herb. 23: 1349-1394.

Standley, P. C. 1934a. Rubiaceae. North American Flora 32(3): 159-228.

Standley, P. C. 1934b. Rubiaceae. North American Flora 32(4): 229-300.

Standley, P. C. \& L. O. Williams. 1975. Rubiaceae. In: Flora of Guatemala. Fieldiana: Botany 24(11): 1-274. 